\title{
A prospective randomized controlled double-blind study comparing auscultation and lung ultrasonography in the assessment of double lumen tube position in elective thoracic surgeries involving one lung ventilation at a tertiary care cancer institute
}

\author{
Swapnil Y Parab, Prashant Kumar, Jigeeshu V. Divatia, and Kailash Sharma \\ Department of Anesthesiology, Critical Care and Pain, Tata Memorial Hospital, Homi Bhabha National Institute, \\ Mumbai, India
}

\begin{abstract}
Background: As lung ultrasound (LUS) can be used to identify regional lung ventilation and collapse, we hypothesize that LUS can be better than auscultation in assessing lung isolation and determining double lumen tube (DLT) position. Methods: A randomized controlled study was conducted in tertiary care cancer institute from November 2014 to December 2015, including 100 adult patients undergoing elective thoracic surgeries. Patients with tracheostomy, difficult airway and pleural-based pathologies were excluded. After anesthesia induction and DLT insertion, patients were randomized into group A (auscultation) and group B (LUS). Regional ventilation was assessed by experienced anesthesiologists using the respective method for each group. Final confirmation of DLT position with a bronchoscope was performed by a blinded anesthesiologist. Contingency tables were plotted to determine sensitivity, specificity, positive predictive value (PPV), negative predictive value (NPV), and accuracy for each method.

Results: Data from 91 patients were analyzed (group A $=47$, group $B=44$ ). Compared with auscultation, LUS had significantly higher sensitivity $(94.1 \%$ vs. $73.3 \%, \mathrm{P}=0.010)$, $\mathrm{PPV}(57.1 \%$ vs. $35.5 \%, \mathrm{P}=0.044)$, NPV $(93.8 \%$ vs. $75.0 \%, \mathrm{P}=$ $0.018)$, accuracy $(70.5 \%$ vs. $48.9 \%, \mathrm{P}=0.036)$ and required longer median time ( $161.5 \mathrm{vs} .114 \mathrm{~s}, \mathrm{P}<0.001)$ for assessment of DLT position. Differences in specificity $(55.6 \%$ vs. $37.5 \%, \mathrm{P}=0.101)$ and area under curve $(0.748$; $95 \%$ CI: $0.604-0.893$ vs. $0.554,95 \%$ CI: $0.379-0.730 ; \mathrm{P}=0.109$ ) were not significant.

Conclusions: Compared to auscultation, LUS is a superior method for assessing lung isolation and determining DLT position.
\end{abstract}

Keywords: Auscultation; Double lumen tube; Lung isolation; Lung ultrasound.

Corresponding author: Swapnil Y Parab, M.D.

Department of Anesthesiology, Critical Care and Pain, Tata Memorial Hospital, Homi Bhabha National Institute, 13, Periyar Building, Anushaktinagar, Mumbai 400094, Maharashtra, India

Tel: 91-9819319866, Fax: 022-24146937, Email: swapnil.parab@gmail.com

ORCID: https://orcid.org/0000-0003-0850-2913

It was presented at 63rd ISACON of Indian Society of Anaesthesiologist, December 2015, B.M. Birla Auditorium \& Convention Centre, Jaipur, India and at 16th World Congress of Anaesthesiologists (WCA), August 2016, Hong Kong Convention and Exhibition Centre, Hong Kong.

Received: December 9, 2017. Revised: June 7, 2018 (1st); August 13, 2018 (2nd); August 30, 2018 (3rd); September 4, 2018 (4th). Accepted: September 4, 2018.

Korean J Anesthesiol 2019 February 72(1): 24-31

https://doi.org/10.4097/kja.d.17.00081

(c) This is an open-access article distributed under the terms of the Creative Commons Attribution Non-Commercial License (http://creativecommons.org/ licenses/by-nc/4.0/), which permits unrestricted non-commercial use, distribution, and reproduction in any medium, provided the original work is properly cited. 


\section{Introduction}

Precise positioning of the double lumen tube (DLT) is a prerequisite for effective lung isolation in thoracic surgeries. Apart from traditional clinical methods such as auscultation, inspection of chest movements, various other methods of confirming DLT position have been developed over the last 3 decades. These methods can be grossly divided into two types: methods that assess DLT position in the trachea (e.g., fiber-optic bronchoscopy [FOB], chest X-ray, fluoroscopy, Trachway ${ }^{\circledR}$ video Stylet, VivaSight ${ }^{\mathrm{TM}}$ Video DLT) and methods that assess regional ventilation and collapse of lung (e.g., auscultation, selective capnography, computerized analysis of breath sounds using microphones, electrical impedance tomography). Both types of methods are used in combination to decide the correct positioning of DLT and their use has been mainly influenced by availability of the instrument and the skills of the operator. Till date, FOB remains the gold standard for confirmation of the DLT position. Among methods that assess the distribution of ventilation in two lungs, auscultation continues to be the prevalent technique, as it is essentially non-invasive and requires basic instruments and skills. However, there is increasing evidence on the inaccuracy of auscultation in assessment of DLT position [1,2].

In last two decades, ultrasonography (USG) has been widely used in various aspects of airway management. Some studies have applied lung ultrasound (LUS) signs like lung sliding (LS) and lung pulse (LP) in assessment of regional distribution of ventilation and have found that LUS is a non-invasive, simple, and effective technique to confirm lung isolation following DLT placement $[3,4]$. In a previous study, we found that addition of LUS improved the diagnostic accuracy of auscultation for the confirmation of left-sided DLT (LDLT) [5]. However, in that study, the surgeon's opinion regarding the quality of lung collapse was used for the final confirmation of DLT position, as FOB was not available during the study period. To overcome this limitation, we designed a prospective randomized controlled study in which FOB was used for the final confirmation of DLT position. Our primary objective was to compare the diagnostic efficacies of auscultation and LUS in the assessment of DLT position. Thus, our primary outcomes were sensitivity, specificity, positive predictive value (PPV), negative predictive value (NPV), and overall accuracy of each method in the assessment of DLT position.

\section{Materials and Methods}

This is a double-blind randomized controlled study conducted at a tertiary care cancer center. Ethical approval for this study was provided by Tata Memorial Hospital's Institutional Ethical Committee on 19 August 2014. The study was registered with Clinical Trial Registry of India (CTRI/2014/11/005198) on 12 November 2014. We screened 130 patients undergoing elective thoracic surgeries and requiring one-lung ventilation (OLV) with the use of DLT for eligibility, out of which 30 were not included in the study (26-failed to meet inclusion criteria, 4declined to participate in the study). Patients below 18 years of age, those with anticipated difficult airways or a tracheostomy tube in situ, or those diagnosed with pneumothorax, pleural effusion or pleurodesis were excluded from the study. One hundred patients were included in the study from 13 November 2014 to 8 December 2015. Patients were screened a day prior to surgery and written informed consent was obtained from those meeting the eligibility criteria. On the day of surgery, patients were randomized to one of the two groups (group A, auscultation [50 patients]; group B, LUS [50 patients]) with the use of a software-generated table of random numbers, prepared by the Institutional Ethical Committee. The group assignment of each patient was placed within sealed envelopes. Envelopes were opened once the patient had been anesthetized, before the assessment of DLT position. Standard 28F, 32F, 35F, 37F and 39F DLTs (Blue Line ${ }^{\circledR}$ Endobronchial Tube, Smiths Medical, Australia) were used. The size and side of DLT were selected according to the judgement of the operation theatre (OT) anesthesiologist. Demographic data and baseline vital parameters of the patient (heart rate, oxygen saturation, blood pressure) were recorded in the case record form (CRF). Following standard anesthesia induction with intravenous injection of propofol $(2-3 \mathrm{mg} / \mathrm{kg})$, fentanyl $(2 \mu \mathrm{g} / \mathrm{kg})$ and rocuronium $(1 \mathrm{mg} / \mathrm{kg})$, the patient's trachea was intubated with a DLT of an appropriate size by OT anesthesiologist. Using a curved McIntosh laryngoscope blade, the DLT with the stylet was passed into the trachea and was turned 90 degrees after the bronchial cuff had passed beyond vocal cords to advance into an appropriate bronchus. The depth of insertion of the DLT at the central incisor level was noted in the CRF. Intra-tracheal placement was confirmed by the end-tidal carbon dioxide $\left(\mathrm{ETCO}_{2}\right)$ monitor. During two-lung ventilation (TLV), the tidal volume was $8 \mathrm{ml} / \mathrm{kg}$ ideal body weight, with the oxygen-air mixture in $1: 1$ ratio. During OLV, tidal volume was $6 \mathrm{ml} / \mathrm{kg}$ with $100 \%$ oxygen. The respiratory rate was adjusted to maintain $\mathrm{ETCO}_{2}$ between 30-35 mmHg. Oxygen saturation was targeted between 93-100\%.

According to the groups assigned, the DLT position was assessed by one of the two methods, i.e., auscultation or LUS. The assessment was carried out in three steps in the supine position. In the first step, the tracheal cuff was inflated to seal leak at the glottis, and both the lungs were ventilated. In the second step, OLV to the corresponding side of the DLT was confirmed by ventilating through the bronchial lumen and inflating the bronchial cuff to seal air entry on the contra-lateral side. In the third step, OLV to the contra-lateral lung corresponding to the DLT was 
confirmed with ventilation through the tracheal lumen. In Group A, regional ventilation was assessed by auscultation performed by an experienced OT anesthesiologist (consultant or senior resident) in each of the above steps. Quality of breath sounds was graded as clear, inconspicuous, or absent. In Group B, regional ventilation was assessed by LUS performed by the principal investigator using USG (M-Turbo ${ }^{\circledR}$ Ultrasound system, SonoSite Ltd., UK) with a 6-13 MHz linear transducer. Ultrasound examination was performed between 2 nd to 4 th inter-costal space in mid-clavicular line and between 5th and 6th inter-costal space in the anterior axillary line. The presence or absence of LP or LS was noted. In both groups, based on the findings, the anesthesiologists were asked to opine whether the DLT position was correct or incorrect. The total time taken for assessment, i.e., the time from placement of stethoscope or ultrasound probe on the patient's chest to the time when the anesthesiologist was ready with an opinion about the DLT position, was also noted in both groups. Following the assessment, the sonography machine and stethoscope were taken out of the OT. A senior, trained anesthesiologist, who was not present in the OT during the assessment of regional ventilation and was blinded to the group assignment, performed FOB (Bronch-Fiberscope $54 \times 2.8^{\circledR}$, Karl Storz, Ger- many) for confirmation of the DLT position. The DLT position was marked as correct if three findings were observed during the FOB examination: (1) upon viewing through the tracheal lumen, there was an unobstructed view of the opposite bronchus; (2) the upper portion of the bronchial cuff was seen just below the carina; (3) upon viewing through the bronchial lumen, the tip of the bronchial tube was well above the secondary carina. If any one of the above three criteria was not met, the DLT position was taken as incorrect. Findings of FOB and the total time taken for the confirmation of DLT position, i.e., from the insertion of FOB to the final decision about DLT position, were noted in the CRF.

Upon bronchoscopic confirmation, if the DLT position was found to be incorrect, then it was corrected under the guidance of FOB. The distance through which DLT was moved inwards or pulled outwards was noted. Repeat FOB was performed after changing the position of the patient, and necessary corrections were made in the DLT position. OLV was started immediately after confirming the DLT position. During OLV, the patient's vital parameters along with peak airway pressures were monitored throughout the surgery. Any intra-operative adverse events like raised peak airway pressure ( $>35 \mathrm{cmH}_{2} \mathrm{O}$ ), episodic drops in oxygen saturation $<92 \%$, application of continuous positive

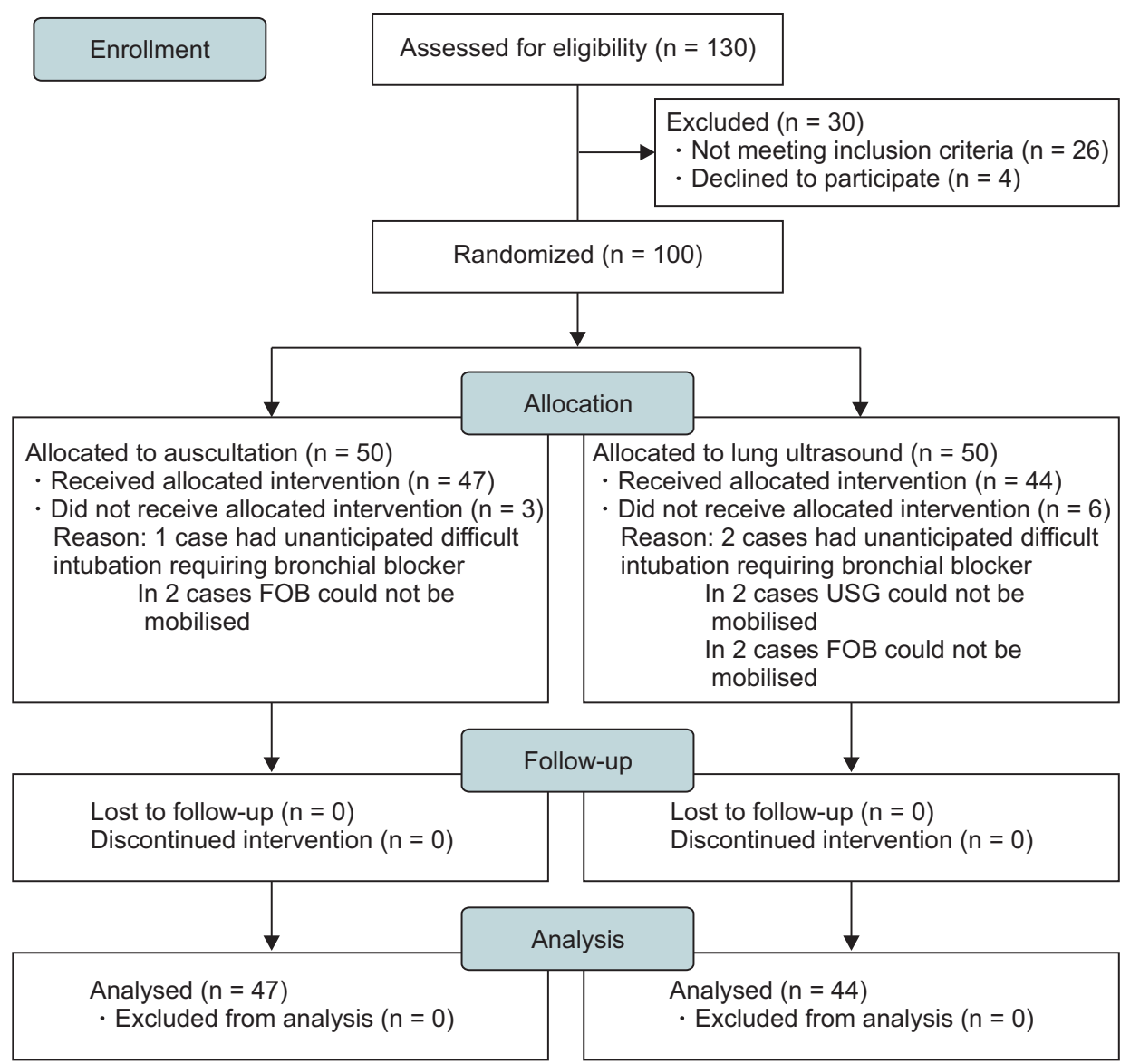

Fig. 1. CONSORT diagram for patient recruitment.

Online access in http://ekja.org 
airway pressure (CPAP), increase in the positive end-expiratory pressure $>5 \mathrm{cmH}_{2} \mathrm{O}$, conversion to TLV were noted. The surgeon's opinion on the lung isolation was noted on a graded scale of excellent (lung fully collapsed), acceptable (lung not fully collapsed but no problems in surgery), and poor (surgeon facing difficulty because of the non-collapsed lung).

Based upon a previously done study by Parab et al. [5], the accuracy of auscultation to detect DLT position was $57 \%$. To test the hypothesis that the use of LUS would improve accuracy to $85 \%$, with an alpha error of 0.05 and power of the study of $80 \%$, the sample size required was 100, i.e., 50 patients in each group. For each method, we defined a true positive (TP), true negative (TN), false positive (FP), and false negative (FN) as follows:

- TP result was defined as a case in which auscultation or LUS suggested a correct DLT position, which was confirmed to be correct after FOB.

- TN result was defined as a case in which auscultation or LUS suggested an incorrect DLT position, which was confirmed to be incorrect after FOB.

- FP result was defined as a case in which auscultation or LUS suggested a correct DLT position, which was found to be incorrect after FOB.

- FN result was defined as a case in which auscultation or LUS suggested an incorrect DLT position, which was found to be correct after FOB.

Sensitivity, specificity, PPV, NPV, and accuracy were calculated as follows:

- Sensitivity $(\%)=\mathrm{TP} /(\mathrm{TP}+\mathrm{FN}) \times 100$

- Specificity $(\%)=\mathrm{TN} /(\mathrm{TN}+\mathrm{FP}) \times 100$

- $\mathrm{PPV}(\%)=\mathrm{TP} /(\mathrm{TP}+\mathrm{FP}) \times 100$

- $\operatorname{NPV}(\%)=\mathrm{TN} /(\mathrm{TN}+\mathrm{FN}) \times 100$

- Accuracy $(\%)=(\mathrm{TP}+\mathrm{TN}) /(\mathrm{TP}+\mathrm{TN}+\mathrm{FP}+\mathrm{FN}) \times 100$.

Sensitivity, specificity, PPV, NPV, and accuracy of the two methods were compared using the $\mathrm{z}$ test for comparing proportions to detect the significance of the difference. Receiver operating characteristic (ROC) graphs were plotted for each group, and areas under curve (AUCs) were compared using the McNeil- Hanley test. Continuous variables were compared using an independent $t$-test, and categorical data were compared using the Chi-square test to find the significance of the differences between the two groups. $\mathrm{P}$ values $<0.05$ were considered statistically significant. For statistics, SPSS (SPSS ${ }^{\circledR}$ Inc., USA) for Windows version 2010 was used.

\section{Results}

Patient enrollment commenced on 13th November 2014. Recruitment of the patients is described in Fig. 1. Baseline patient characteristics in both groups were comparable (Table 1).

Analysis and comparison of the contingency table (Table 2) showed that the sensitivity ( $94.1 \%$ vs. $73.3 \%, \mathrm{P}=0.010)$, PPV (57.1\% vs. $35.5 \%, \mathrm{P}=0.044)$, NPV (93.8\% vs. $75.0 \%, \mathrm{P}=0.018$ ) and accuracy $(70.5 \%$ vs. $48.9 \%, \mathrm{P}=0.036)$ of group $\mathrm{B}$ were significantly higher than those of group A (Table 3 ). Specificity of group B was better than that of group A but this improvement was not significant (55.6\% vs. $37.5 \%, \mathrm{P}=0.101)$. ROC curves were plotted (Fig. 2), which showed that the AUC for group B was higher than that for group A (0.748 vs. 0.554$)$, but this difference was not significant $(\mathrm{P}=0.109)$.

Out of the 91 patients considered for analysis, in 32 patients (group A, 15 patients; group B, 17 patients), there was no further adjustment required in the DLT position after FOB, and these DLTs were correctly placed. In the remaining 59 patients (group A, 32 patients; group B, 27 patients), some repositioning was required after FOB, and hence, the DLTs were considered to be incorrectly placed. Further analysis of these 59 patients, as per the classification suggested by Cohen, is summarized in Table 4 [6]. In both groups, $100 \%$ accuracy in the identification of critically mal-positioned tubes was noted.

Table 1. Baseline Patient Characteristics

\begin{tabular}{|c|c|c|c|}
\hline Parameters & $\begin{array}{c}\text { Group A } \\
(\mathrm{n}=47)\end{array}$ & $\begin{array}{l}\text { Group B } \\
(\mathrm{n}=44)\end{array}$ & $P$ value \\
\hline Age (yr) & $52.2 \pm 11.9$ & $48.8 \pm 14.5$ & 0.294 \\
\hline $\operatorname{Sex}(M / F)$ & $39 / 8$ & $30 / 14$ & 0.099 \\
\hline Height $(\mathrm{cm})$ & $164.4 \pm 7.0$ & $164.2 \pm 10.0$ & 0.726 \\
\hline Weight (kg) & $62.8 \pm 10.8$ & $61.7 \pm 11.8$ & 0.617 \\
\hline $\operatorname{BMI}\left(\mathrm{kg} / \mathrm{m}^{2}\right)$ & $23.1 \pm 3.1$ & $22.8 \pm 3.9$ & 0.335 \\
\hline ASA-PS & & & 0.325 \\
\hline I & 27 & 29 & \\
\hline II & 18 & 15 & \\
\hline III & 2 & 0 & \\
\hline Types of surgeries & & & 0.900 \\
\hline Lung resection surgeries & 31 & 27 & \\
\hline Esophageal & 11 & 12 & \\
\hline Other surgeries & 5 & 5 & \\
\hline Surgical approach & & & 0.394 \\
\hline Robotic & 11 & 7 & \\
\hline VATS & 13 & 18 & \\
\hline Open & 23 & 19 & \\
\hline Size of DLTs inserted & & & 0.134 \\
\hline $28 \mathrm{~F}$ & 1 & 1 & \\
\hline $32 \mathrm{~F}$ & 0 & 1 & \\
\hline $35 \mathrm{~F}$ & 5 & 10 & \\
\hline $37 \mathrm{~F}$ & 28 & 15 & \\
\hline $39 \mathrm{~F}$ & 13 & 17 & \\
\hline Side of DLT used & & & 0.608 \\
\hline Left DLT & 46 & 42 & \\
\hline Right DLT & 1 & 2 & \\
\hline
\end{tabular}

Values are expressed as mean \pm SD or number of patients. Group A: auscultation group, Group B: lung ultrasound group. BMI: body mass index, ASA-PS: American Society of Anesthesiologists physical status, DLT: double lumen tube, VATS: video-assisted thoracoscopic surgery. 
Table 2. Contingency Table for Groups A and B

\begin{tabular}{clccc}
\hline \multirow{2}{*}{ Groups } & & \multicolumn{2}{c}{$\begin{array}{c}\text { Opinion on DLT } \\
\text { position by FOB }\end{array}$} & $\begin{array}{c}\text { Total } \\
(\mathrm{n}=91)\end{array}$ \\
\cline { 3 - 4 } & & Correct & Incorrect & \\
\hline \multirow{2}{*}{ Group A } & Correct & 11 & 20 & $\mathrm{n}=47$ \\
& Incorrect & 4 & 12 & \\
\multirow{2}{*}{ Group B } & Correct & 16 & 12 & $\mathrm{n}=44$ \\
& Incorrect & 1 & 15 & \\
\hline
\end{tabular}

Group A: auscultation group, Group B: lung ultrasound groups. DLT: double lumen tube, FOB: fiberoptic bronchoscopy.

\section{Group A - Auscultation}

ROC curve

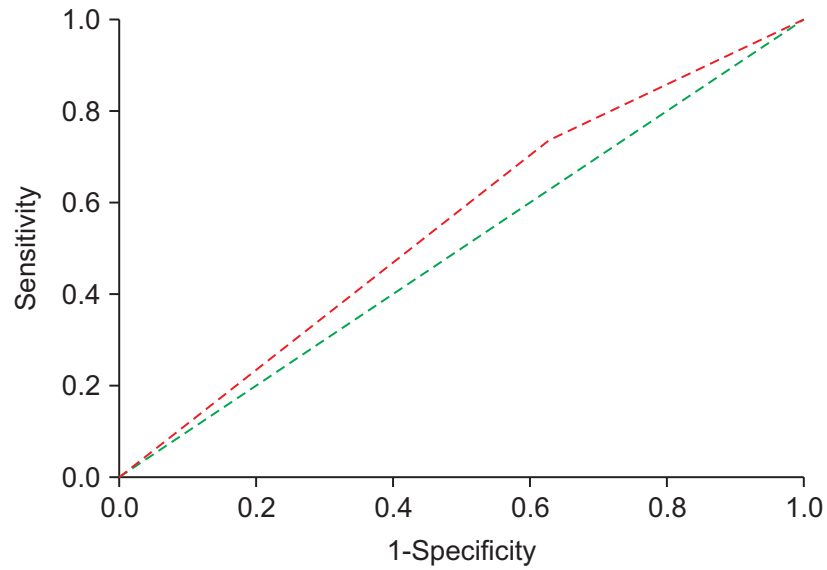

Diagonal segments are produced by ties.
Table 3. Comparison of Primary Outcomes between Groups A and B

\begin{tabular}{lccccccc}
\hline \multirow{2}{*}{$\begin{array}{c}\text { Primary } \\
\text { outcome }\end{array}$} & \multicolumn{2}{c}{ Group A (n= 47) } & & \multicolumn{3}{c}{ Group B $(\mathrm{n}=44)$} & \\
\cline { 2 - 3 } & Value (\%) & $95 \%$ CI & & Value (\%) & $95 \%$ CI & \\
\hline Sensitivity & 73.3 & $44.8-91.1$ & & 94.1 & $69.2-99.7$ & 0.010 \\
Specificity & 37.5 & $21.7-56.3$ & & 55.6 & $35.6-74.0$ & 0.101 \\
PPV & 35.5 & $19.8-54.6$ & & 57.1 & $37.4-75.0$ & 0.044 \\
NPV & 75.0 & $47.0-91.7$ & & 93.8 & $67.7-99.7$ & 0.018 \\
Accuracy & 48.9 & $34.7-62.5$ & & 70.5 & $54.8-83.2$ & 0.036 \\
\hline
\end{tabular}

Group A: auscultation group, Group B: lung ultrasound group. PPV: positive predictive value, NPV: negative predictive value.

\section{Group B - Lung ultrasound}

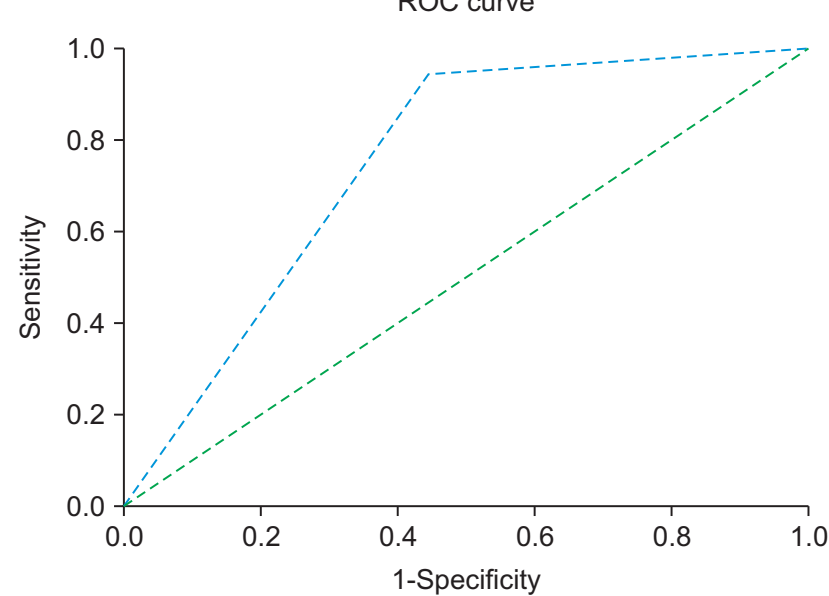

Diagonal segments are produced by ties.

Fig. 2. Receiver operating characteristic (ROC) curves for the two groups. Area under curve (AUC) for group B (0.748 with standard error $0.074 ; 95 \%$ $\mathrm{CI}, 0.604-0.893)$ was higher than AUC for group A (0.554 with standard error 0.089, 95\% CI, 0.379-0.730), but this difference was not significant $(\mathrm{P}=$ $0.109)$.

Three right-sided DLTs (RDLTs) were used during the study: two in group B and one in group A. The position of all these DLTs was interpreted correctly by the randomized method. The median time for the assessment for group B (161.5 s; range, 64-360 s) was significantly higher than that required for group A (114 s; range, 60-300 s) ( $<$ < 0.001). Patient intra-operative and post-operative outcomes are summarized in Table 5.

\section{Discussion}

A thoracic anesthesiologist primarily needs to know two things about the position of the DLT when it is being used for the surgical exposure. The first is the precise position of the DLT in the tracheo-bronchial tree, best confirmed by FOB, which assures the anatomical separation of the lungs; the second is confirmation of the distribution of regional ventilation, i.e., whether the lung on the operative site is collapsed and whether the other lung is being adequately ventilated, which can be considered as functional isolation and is commonly assessed by auscultation. Newer methods like LUS or electrical impedance tomography have been used to assess regional ventilation. In this study, we found that LUS is more reliable and accurate than auscultation for the assessment of functional isolation and thereby for the assessment of the DLT position. Alliaume et al. [7] found that auscultation is not reliable for confirmation of the DLT position and FOB was required for $78 \%$ LDLTs and $83 \%$ RDLTs for proper placement. de Bellis et al. [2] also found that 32\% of DLTs shown to be correctly placed by auscultation had to be repositioned after FOB and 5\% of DLTs were critically mal-positioned. Possible reasons for this inaccuracy could be that the audibility of breath sounds is often affected by tidal volume, consistency of underlying lung tissue, thickness of the chest wall, noise intensity in the OT, sensitivity of the stethoscope, and hearing acuity of the individual. Auscultation of one side of the chest is also confounded by the conductance of sound from the other side of the chest. As a result, auscultation is often not relied upon in 
Table 4. Analysis of DLTs that Required Repositioning after FOB

\begin{tabular}{|c|c|c|c|}
\hline $\begin{array}{l}\text { Classification as per degree of } \\
\text { repositioning of DLT }\end{array}$ & Group A & Group B & Total \\
\hline $\begin{array}{l}\text { Optimally placed (repositioning } \\
\text { by less than or equal to } 0.5 \mathrm{~cm} \text { ) }\end{array}$ & 7 & 5 & 12 \\
\hline $\begin{array}{l}\text { Misplaced (repositioning by more } \\
\text { than } 0.5 \mathrm{~cm} \text { ) }\end{array}$ & 19 & 16 & 35 \\
\hline $\begin{array}{l}\text { Critically misplaced (DLT removed } \\
\text { and re-inserted or changed to } \\
\text { bronchial blocker) }\end{array}$ & 6 & 6 & 12 \\
\hline Total & 32 & 27 & 59 \\
\hline
\end{tabular}

Values are expressed as number of patients. Group A: auscultation group, Group B: lung ultrasound group. DLT: double lumen tube, FOB: fiberoptic bronchoscopy.

theaters where FOB facilities are available. However, when FOB is not available, auscultation is the most commonly used non-invasive technique to assess the distribution of ventilation.

In the recent times, USG has received wider acceptability in critical care settings and increasing applicability in various aspects of airway management in OTs. It is a non-invasive technique that provides a visual image that can be shared simultaneously, and even remotely, and can preserve images in a digital format allowing for accurate documentation and medical education. USG has been identified as a useful tool for the confirmation of the correct position of the endotracheal tube [8-10]. On ventilation of a lung, LS can be identified immediately at the pleural interface. As soon as the ventilation is stopped, the sliding is abolished, and pulsatile movement is apparent at the pleural interface. This pulsatile movement is called a 'LP', which has been demonstrated to have $93 \%$ sensitivity and $100 \%$ specificity as a sign for the identification of lung collapse [11]. Thus, if USG can demonstrate LS on one side and a LP on the other side, then functional isolation can be assured and correct DLT position can be assumed. The accuracy of this can be further improved by the demonstration of a power sliding sign using color Doppler [12]. This potential application of LUS is further supported by findings of a few other studies.

Šustić et al. [3], in a prospective randomized study, found that the addition of a brief ultrasound examination to clinical assessment improved specificity ( $50 \%$ vs. $22 \%$ ), accuracy ( $88 \%$ vs. $72 \%$ ), and PPV ( $86 \%$ vs. $70 \%$ ) of clinical assessment for the detection of correct position of LDLT. Álvarez-Díaz et al. [4], in a crossover study, compared the transthoracic LUS and clinical methods for confirmation of LDLT position in 105 patients and found that LUS was superior to clinical methods in terms of sensitivity ( $98.6 \%$ vs. $84.5 \%$ ), specificity (52.9\% vs. $41.1 \%)$, accuracy ( $83.8 \%$ vs. $70.4 \%$ ), PPV ( $81.4 \%$ vs. $75.0 \%$ ), and NPV ( $94.7 \%$ vs. $56.0 \%)$. Earlier, we performed a prospective comparative study in our institute and found that, compared to the use of clinical methods alone, addition of lung USG improved sensitivity $(75 \%$
Table 5. Summary of Patient Outcomes

\begin{tabular}{|c|c|c|}
\hline Outcome parameter & Group A & Group B \\
\hline \multicolumn{3}{|l|}{$\begin{array}{l}\text { Quality of lung isolation as per the opinion } \\
\text { of the surgeons }(n=87)\end{array}$} \\
\hline Excellent & 36 & 38 \\
\hline Acceptable & 8 & 4 \\
\hline Poor & 0 & 1 \\
\hline \multicolumn{3}{|l|}{ Intra-operative complications $(\mathrm{n}=38)$} \\
\hline Raised airway pressure & 8 & 3 \\
\hline $\begin{array}{l}\text { Episodes of drop in oxygen saturation below } \\
92 \%\end{array}$ & 5 & 7 \\
\hline $\begin{array}{l}\text { Both raised airway pressure and drop in } \\
\text { oxygen saturation below } 92 \%\end{array}$ & 4 & 5 \\
\hline VATS surgery converted to open thoracotomy & 2 & 3 \\
\hline Hemodynamic instability & 0 & 1 \\
\hline \multicolumn{3}{|l|}{ Intra-operative interventions $(\mathrm{n}=12)$} \\
\hline CPAP to the non-dependent lung & 0 & 2 \\
\hline $\mathrm{PEEP}>5 \mathrm{cmH}_{2} \mathrm{O}$ & 5 & 3 \\
\hline $\begin{array}{l}\text { Both CPAP to nondependent lung and PEEP } \\
>5 \mathrm{cmH}_{2} \mathrm{O}\end{array}$ & 0 & 1 \\
\hline Bronchoscopic tube repositioning & 0 & 1 \\
\hline Post-operative ventilation & 0 & 4 \\
\hline In hospital mortality & 0 & 4 \\
\hline
\end{tabular}

Values are expressed as number of patients. Group A: auscultation group, Group B: lung ultrasound group. OLV: one lung ventilation, DLT: double lumen tube, VATS: video-assisted thoracoscopic surgery, CPAP: continuous positive airway pressure, PEEP: positive end-expiratory pressure.

vs. $88 \%$ ), specificity ( $18 \%$ vs. $75 \%)$, and accuracy ( $57 \%$ vs. $85 \%)$ for correct prediction of LDLT position [5]. However, a major limitation of the study was that FOB was not used for the final confirmation. In addition, the patients were not randomized into two groups. We overcame these limitations in the present study. With applications of stringent bronchoscopic parameters for defining the correct DLT position, we found that the accuracy of auscultation decreased further (57\% in the previous study vs. $48.9 \%$ in the present study). Although the accuracy of LUS also decreased ( $85 \%$ in the previous study vs. $70.5 \%$ in the present study), it is still significantly higher than that of auscultation. Nam et al. [13] reported the use of LUS to confirm lung isolation in an infant. Using LUS, the authors could achieve successful positioning of the endobronchial tube, when a skilled bronchoscopy operator was not available in the emergency setting. Thus, LUS is not only superior but also a more reliable technique than auscultation, as far as the assessment of lung isolation is considered. With an increasing number of applications in the field of perioperative care, USG machine is increasingly becoming a cost-effective alternative for the institutes to invest in.

LUS has often been criticized as it necessitates an anesthesiologist to acquire an additional skill. However, Lyon et al. [14] tested the utility of LUS in the hands of the pre-hospital critical care providers and showed that they can accurately determine 
the presence or absence of the sonographic LS sign following a brief tutorial and can retain the skill even after 9 months. Saporito et al. [15] showed that LUS, in the hands of a trained nurse anesthetist, could be not only as specific and sensitive but also more rapid and cost-effective than FOB in confirming the position of LDLT. Both these studies highlight that LUS skills are simple to learn and retain. However, in our opinion, LUS cannot be an alternative to FOB. There are some distinct applications of FOB where LUS has no role. For example, the adjustment of RDLT to facilitate right upper lobe ventilation and intra-operative adjustment of DLT can be made only with FOB. Thus, FOB would continue to be the gold standard method for determining the precise position of a DLT but, owing to its ability to confirm functional lung isolation, LUS is a better complimentary method to FOB than auscultation.

Finally, it is worth noting the disadvantages of LUS. Besides the availability and cost of the machine, the possibility of inter-individual variation exists. A study by Ponsonnard et al. [16] has shown the utility of LUS in predicting right upper lobe atelectasis and concluded that LUS is not so relevant in RDLT positioning. In our study, only two RDLT cases were randomized to the LUS group and the positions in both were identified correctly by LUS. However, due to a smaller number of cases, no inference could be drawn. LUS is not reliable in cases where precise positioning of DLT and an airtight seal is mandatory, like in bronchoalveolar lavage or massive hemoptysis. In addition, any condition which can minimize the movement of two layers of pleura against each other can abolish the LS sign. For example, in the patients with surgical pleurodesis or post inflammatory pleural adhesions (e.g., in the damaged lung following pleuro-pulmonary tuberculosis) or mesothelioma, pleural signs would be abolished. During the exacerbation of chronic obstructive pulmonary disease, which may result in the hyperinflation of the lung, the signs of lung sliding may be absent. Such conditions must be taken into account before using LUS.

Further, the limitations of our study must be acknowledged. A crossover study design would have been better to avoid any patient- or observer-related bias in the study. To minimize the inter-investigator variation, all LUS examinations were performed by the same investigator throughout the study; this may overestimate the accuracy of the results, particularly with the investigator gaining experience through the course of the study. Although our desired sample size was 100 patients, we could include only 91 patients for the final analysis. Thus, the study is slightly underpowered. Furthermore, we did not keep a visual record of the LS and LP sign for each patient. The patients did not receive a fixed tidal volume during the assessment of the DL position in both groups. As the patients received manual ventilation during the assessment of DLT position, the conductance of sound may vary with each breath owing to varying tidal volumes.

In conclusion, this study shows that LUS significantly improves the accuracy in the assessment of the DLT position. LUS has better sensitivity, specificity, PPV, and NPV for confirmation of lung isolation and is a better complimentary technique for FOB than auscultation.

\section{ORCID}

Swapnil Y Parab, https://orcid.org/0000-0003-0850-2913

Prashant Kumar, https://orcid.org/0000-0001-7091-8288

Jigeeshu V. Divatia, https://orcid.org/0000-0001-7384-4886

\section{References}

1. Smith GB, Hirsch NP, Ehrenwerth J. Placement of double-lumen endobronchial tubes. Correlation between clinical impressions and bronchoscopic findings. Br J Anaesth 1986; 58: 1317-20.

2. de Bellis M, Accardo R, Di Maio M, La Manna C, Rossi GB, Pace MC, et al. Is flexible bronchoscopy necessary to confirm the position of double-lumen tubes before thoracic surgery? Eur J Cardiothorac Surg 2011; 40: 912-6.

3. Sustić A, Protić A, Cicvarić T, Zupan Z. The addition of a brief ultrasound examination to clinical assessment increases the ability to confirm placement of double-lumen endotracheal tubes. J Clin Anesth 2010; 22: 246-9.

4. Álvarez-Díaz N, Amador-García I, Fuentes-Hernández M, Dorta-Guerra R. Comparison between transthoracic lung ultrasound and a clinical method in confirming the position of double-lumen tube in thoracic anaesthesia. A pilot study. Rev Esp Anestesiol Reanim 2015; 62: 305-12.

5. Parab SY, Divatia JV, Chogle A. A prospective comparative study to evaluate the utility of lung ultrasonography to improve the accuracy of traditional clinical methods to confirm position of left sided double lumen tube in elective thoracic surgeries. Indian J Anaesth 2015; 59: 476-81.

6. Cohen E. Double-lumen tube position should be confirmed by fiberoptic bronchoscopy. Curr Opin Anaesthesiol 2004; 17: 1-6.

7. Alliaume B, Coddens J, Deloof T. Reliability of auscultation in positioning of double-lumen endobronchial tubes. Can J Anaesth 1992; 39: 687-90.

8. Das SK, Choupoo NS, Haldar R, Lahkar A. Transtracheal ultrasound for verification of endotracheal tube placement: a systematic review 
and meta-analysis. Can J Anaesth 2015; 62: 413-23.

9. Blaivas M, Tsung JW. Point-of-care sonographic detection of left endobronchial main stem intubation and obstruction versus endotracheal intubation. J Ultrasound Med 2008; 27: 785-9.

10. Pfeiffer P, Rudolph SS, Børglum J, Isbye DL. Temporal comparison of ultrasound vs. auscultation and capnography in verification of endotracheal tube placement. Acta Anaesthesiol Scand 2011; 55: 1190-5.

11. Lichtenstein DA, Lascols N, Prin S, Mezière G. The "lung pulse": an early ultrasound sign of complete atelectasis. Intensive Care Med 2003; 29: 2187-92.

12. Cunningham J, Kirkpatrick AW, Nicolaou S, Liu D, Hamilton DR, Lawless B, et al. Enhanced recognition of "lung sliding" with power color Doppler imaging in the diagnosis of pneumothorax. J Trauma 2002; 52: 769-71.

13. Nam JS, Park I, Seo H, Min HG. The use of lung ultrasonography to confirm lung isolation in an infant who underwent emergent videoassisted thoracoscopic surgery: a case report. Korean J Anesthesiol 2015; 68: 411-4.

14. Lyon M, Walton P, Bhalla V, Shiver SA. Ultrasound detection of the sliding lung sign by prehospital critical care providers. Am J Emerg Med 2012; 30: 485-8.

15. Saporito A, Lo Piccolo A, Franceschini D, Tomasetti R, Anselmi L. Thoracic ultrasound confirmation of correct lung exclusion before onelung ventilation during thoracic surgery. J Ultrasound 2013; 16: 195-9.

16. Ponsonnard S, Karoutsos S, Gardet E, Marsaud JP, Nathan N. Value of lung sonography to control right-sided double lumen endotracheal tube location. J Anesth Clin Res 2014; 5: 1000453. 